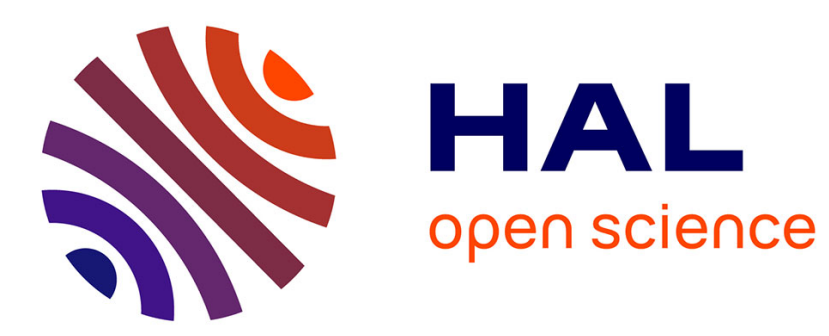

\title{
De la nécessité d'adopter une posture scientifique et critique au temps du numérique
}

Cécile Dolbeau-Bandin, Serge Proulx, Vassili Rivron

\section{To cite this version:}

Cécile Dolbeau-Bandin, Serge Proulx, Vassili Rivron. De la nécessité d'adopter une posture scientifique et critique au temps du numérique. Terminal. Technologie de l'information, culture \& société, 2016, Us et abus de l'Internet, 119, [15 p.]. 10.4000/terminal.1512 . hal-01938637

\section{HAL Id: hal-01938637}

\section{https://hal-normandie-univ.archives-ouvertes.fr/hal-01938637}

Submitted on 28 Nov 2018

HAL is a multi-disciplinary open access archive for the deposit and dissemination of scientific research documents, whether they are published or not. The documents may come from teaching and research institutions in France or abroad, or from public or private research centers.
L'archive ouverte pluridisciplinaire $\mathbf{H A L}$, est destinée au dépôt et à la diffusion de documents scientifiques de niveau recherche, publiés ou non, émanant des établissements d'enseignement et de recherche français ou étrangers, des laboratoires publics ou privés. 


\section{Terminal}

Technologie de l'information, culture \& société

$119 \mid 2016$

Us et abus de l'Internet

\section{De la nécessité d'adopter une posture scientifique} et critique au temps du numérique

The need to adopt a scientific and critical attitude in the digital age

Cécile Dolbeau-Bandin, Serge Proulx et Vassili Rivron

\section{revues.org}

Éditeur

CREIS-Terminal

Édition électronique

URL : http://terminal.revues.org/1512

ISSN : 2429-4578

Référence électronique

Ce document a été généré automatiquement le 8 janvier 2017. 


\title{
De la nécessité d'adopter une posture scientifique et critique au temps du numérique
}

The need to adopt a scientific and critical attitude in the digital age

\author{
Cécile Dolbeau-Bandin, Serge Proulx et Vassili Rivron
}

\section{NOTE DE L'ÉDITEUR}

L'ensemble des textes a été harmonisé par C. Dolbeau-Bandin et V. Rivron.

1 Le déploiement de l'infrastructure de l'Internet, la multiplication croissante des objets qui y sont branchés et la profusion des pratiques sociales qui en dépend désormais, mènent souvent à évoquer l'idée d'un tournant civilisationnel majeur, une révolution, au même titre que l'ont été l'avènement de l'écriture ou de l'imprimerie. Il est ainsi devenu commun de parler d'une "ère Internet " ou d'un "âge numérique ", car des domaines essentiels de la vie sociale semblent devoir être repensés depuis les formes et les rythmes de travail jusqu'aux relations interpersonnelles et affectives, en passant par les formes de médiation culturelle, d'organisation et de participation à la vie publique. C'est ce qui conforte le sentiment partagé d'une nouvelle «Grande Transformation» [Moulier Boutang, 2007 en référence à Polanyi, 1944] qui érige une autonomie nouvelle de la sphère informationnelle, ayant des effets depuis les infrastructures sociales, politiques, économiques jusqu'aux subjectivités.

2 Les capacités nouvelles de collecte et de traitement de l'information font aussi basculer des pans entiers des pratiques, objets et méthodes scientifiques suscitant des prises de position sur cet Internet que l'on ne peut plus réduire à un simple "épiphénomène social ». Ce qui nous préoccupe dans le cadre de ce dossier de Terminal et qui donne suite à la journée d'étude "Us et abus d'Internet : enjeux sociaux et psychologiques des pratiques numériques $»^{1}$, est la volonté de dépasser les antinomies construites par le 
marketing, par le jeu politique ou cristallisées dans le sens commun. De même que la rhétorique des «usages" peut participer des discours enthousiastes prescrivant l'innovation comme valeur positive en soi [Guichard, 2012], l'idée d'«abus du numérique » fait partie d'un discours « dystopique » tout aussi réducteur, qui annonce au contraire, pertes de repères, mondes bousculés, ruptures générationnelles, peurs... Il s'agit ici au contraire de considérer ensemble ce qui est perçu alternativement comme des opportunités ou des dérives induites par les usages des technologies numériques, et d'analyser ses effets en termes de mouvement structurant et structuré.

3 À plus d'un titre, ces questions bousculent les chercheurs en sciences humaines et sociales. Et en particulier, elles soulèvent des questions à la fois normatives et réflexives. D'une part, où et comment pouvons-nous placer le curseur entre us et abus? Les nouvelles configurations sociales engendrées dans l'autre environnement sociotechnique font-elles place à des formes de relations asymétriques, liées notamment aux abus de certaines plateformes envers les usagers?

4 La pénétration massive de l'informatique à partir des années 1980, puis de l'Internet à partir du milieu des années 1990 (en France) contraste avec le réveil tardif d'une réflexivité critique sur les effets (sans déterminisme technologique) de l'introduction de ces techniques dans des univers sociaux déjà constitués, mais du fait des propriétés qui lui sont spécifiques, des agents sociaux qui les portent et de ceux qui se les approprient.

5 Face à ces mutations importantes et aux discours engagés qui les accompagnent, nous avons souhaité réunir des spécialistes de différentes disciplines en sciences humaines et sociales (SHS) afin de mettre en rapport des visions distancées et d'évaluer de façon critique ces mutations civilisationnelles, au sens de Norbert Elias (1973). Un tournant critique s'annonce ces dernières années dans les sciences sociales de l'informatique connectée, prenant ses distances avec les enthousiastes de la table rase et l'embrigadement dans les sciences appliquées [Guichard, 2012 ; Matthews, 2010 ; Granjon, 2015]. Au contraire, même les sciences informatiques qui s'emparent de questions sociétales dans une logique appliquée demandent aujourd'hui à s'approprier plus rigoureusement des traditions critiques en sciences sociales ${ }^{2}$. Cette posture critique devrait à la fois :

- être neutre, dépassionnée, mobilisant l'ancrage social des théories et représentations sur le numérique ;

- renouer avec les traditions critiques et les postures épistémologiques progressivement élaborées en SHS au cours des derniers siècles;

- n'être ni invérifiable ni purement inductive ;

- se méfier de l'illusion de l'exhaustivité du big data [Anderson, 2008; Cardon, 2015], une erreur dans laquelle les sciences sociales sont déjà tombées ;

- être à la fois appliquée et critique, dans le sens où elle révèle aux acteurs la construction sociale des enjeux de leurs champs d'action. Elle est nécessaire pour contribuer à positionner le curseur entre us et abus, pour objectiver les mutations des rapports sociaux, pour désenchanter certains discours mobilisateurs qui ne servent pas l'intérêt général, pour repenser les idéaux politiques qui animent la plupart des jugements portés sur la technique ;

- rendre la réalité inacceptable [Boltanski, 2008].

6 Les sciences critiques doivent ainsi s'interroger sur le bien-fondé et les implications de toutes les "promesses ", "solutions » et «opportunités " offertes par les porteurs de projets, services et matériels (par exemple, revisiter de façon critique les 21 promesses du numérique recensées par la Fondation Internet Nouvelle Génération ou Fing, dans 
Questions numériques, 2013-2014). En effet, les technologies numériques seraient-elles susceptibles de résoudre des problèmes déjà bien connus (les domaines éducatif, énergétique, l'exclusion sociale, etc.) ? Comment penser la " puissance d'agir citoyenne » qui se manifeste dans bien des secteurs supportés par l'informatique connectée, face à une société de contrôle et de surveillance dominée par des formes d'hégémonie inédites (pensons à l'emprise des Gafa - Google, Apple, Facebook, Amazon) ? L'appréhension éthique de ces modes de sociabilité à l'ère des médiations technologiques s'avère-t-elle particulièrement ouverte ? Est-il possible de prendre la pleine mesure de l'ambiguïté des pratiques numériques, située au croisement d'un risque de dépendance à la technique et de phénomènes de résistance culturelle? Les discours médiatiques habituels sur les addictions et les dépendances sont-ils toujours définis clairement ? Ces deux concepts ne sont-ils pas parfois confondus ? L'ordinateur connecté est-il un support facilitant l'accès au jeu pour des joueurs pathologiques? Internet comporte-t-il des caractéristiques jouant un rôle important dans l'exacerbation de pratiques de jeu excessives? Comment les individus s'approprient-ils dans leur vie quotidienne les médias traditionnels (presse, radio, télévision) en conjonction avec les nouveaux médias numériques (agrégateurs de contenus, sites d'information en ligne, blogs d'experts ou d'amateurs) ? Quels sont les usages « culturels» de l'Internet, et par cet exemple restreint, comment rendre compte de l'importance de la culture numérique dans les enjeux citoyens?

7 Cette démarche n'offre pas de légitimation facile au développement des industries du numérique, mais elle offre une lecture critique des pratiques individuelles et collectives. Il nous a semblé salutaire d'opposer des contre-feux aux «Assises du numérique » qui avaient lieu à Caen pendant la même période, et qui prennent le numérique comme du "pain béni » pour des politiques publiques et en particulier éducatives, informées principalement par les lobbys industriels et les technophiles enthousiastes. L'objectif de cette journée d'étude a été d'analyser, dans une perspective pluridisciplinaire, les risques et les vulnérabilités individuels et/ou collectifs dans les sociétés contemporaines, qu'elles soient occidentales ou non, concernées par les usages du numérique.

8 Les techniques de l'informatique connectée se sont désormais déployées dans la plupart des secteurs d'activité et bouleversent à divers degrés [Tisseron, 2012] nos habitudes, nos savoir-faire (lire, écrire, raconter, travailler, jouer), interroge nos certitudes et par là même l'ensemble de nos paradigmes économiques, juridiques, technologiques et sociétaux. Les transformations engendrées sont d'une ampleur et d'une complexité telle, qu'il n'a pas été évident pour les sciences humaines et sociales de développer une réflexion structurée capable de les comprendre. Cette société numérique dans laquelle nous évoluons aujourd'hui et dans laquelle nous vivrons demain, promet d'être une aventure à la fois «fascinante et effrayante », pour emprunter l'expression aux penseurs de l'École de Francfort.

9 Tout au long de la réflexion collective qui nous a réunis lors de la journée d'étude et le présent dossier, les participants ont pu constater la grande diversité des approches mises en œuvre par les auteurs des textes. Cette diversité concerne aussi bien les disciplines mobilisées (sciences de l'information et de la communication, philosophie, psychanalyse, psychiatrie, sociologie de l'innovation, sociologie des médias) que les modèles théoriques de référence (sociologie des usages et des cultures numériques, éthique de la technique, psychologie culturelle et psychopathologie du jeu), les territoires considérés (régional, national, international) ou les méthodologies utilisées (de la monographie à la 
spéculation théorique, en passant par les enquêtes quantitatives, les approches qualitatives et les analyses des représentations).

Cette diversité méthodologique n'a pas empêché les intervenants de mettre en résonance trois niveaux de convergence entre leurs propositions. Tout d'abord une toile de fond épistémologique globalement partagée, à quelques variations près. Ce choix commun s'est traduit par une mise à distance des discours idéologiques sur Internet. Ce qui les conduit à une vision nuancée et prudente des pratiques numériques. Même si certains ont penché davantage pour une approche critique et d'autres vers une approche évaluative, voire thérapeutique, cela s'est fait avec beaucoup de précautions rhétoriques et méthodologiques.

11 Les discussions de la journée d'étude se sont organisées en quatre séquences thématiques, que nous reprenons dans le présent dossier de Terminal, en rappelant les principales interventions de la journée d'étude et en introduisant les textes choisis qui constituent ce numéro :

- Connectivité : usages participatifs et espace public ;

- Éthique et humanités numériques ;

- Addictions et culture des écrans ;

- Information et accès aux savoirs.

\section{Connectivité : usages participatifs et espace public}

Internet a suscité de nouvelles formes de participation à la vie publique et repoussé les frontières des idéaux démocratiques prégnants dans les sociétés occidentales [Cardon, 2010]. Le renouvellement lexical et sémantique que le dispositif sociotechnique suscite, oriente le perfectionnement technologique de nos sociétés vers des objectifs affirmés visant plus d'égalité et plus de liberté, voire plus de fraternité (accessibilité, neutralité, gratuité, communauté, partage). Ne définissant pas clairement les droits d'entrée (majorité, nationalité, compétence, coût), les projets participatifs réactivent à la fois le modèle antique de la démocratie directe et l'imaginaire fin XIXe d'une politique de la foule (s'exprimant aujourd'hui à travers les notions de crowdsourcing ${ }^{3}$, crowdfunding ${ }^{4}$, crowdsensing $\left.{ }^{5} ..\right)$ tout en questionnant l'organisation en culture de masse de l'espace public bourgeois [Habermas, 1962].

Mais de quelle démocratie parle-t-on aujourd'hui? Nous sommes devant une masse au périmètre indéfini, qui ignore ceux qui ne sont pas connectés aux différentes plateformes dont on ne sait pas encore produire une représentativité, ce qui ne construit pas, précisément, un espace public. $\mathrm{Si}$, comme nous l'a rappelé Évelyne Broudoux ${ }^{6}$, l'informatique connectée promeut de nouvelles modalités relationnelles à distance (coopération, collaboration, participation), il faut encore s'interroger sur les rapports politiques que cela implique, en rétablissant les acteurs et les processus concernés par ces dispositifs. En effet, trop souvent la rhétorique de la désintermédiation et de la dématérialisation tend à présenter ces nouvelles techniques comme neutres et transparentes. Or la démocratie représentative passe déjà par des dispositifs techniques de production de l'opinion publique (presse, sondages, isoloirs) qui ont fait l'objet de maintes discussions. Il faut maintenant s'intéresser aux spécificités de ces nouveaux dispositifs d'expression et de coordination politique - sans jamais oublier ceux qui les portent et parfois, leur engagement subjectif vis-à-vis d'un certain « désintéressement » 
[Bourdieu, 1994] - et en particulier, par la mise à disposition de services apparemment gratuits [Revue du Mauss, 2010].

Évelyne Broudoux attire également l'attention sur les contradictions fortes entre le caractère magique des promesses du progrès technologique et l'évaluation de leurs effets observables, notamment à travers l'analyse des projets regroupés sous le label smart cities $^{7}$, une visée utopique promue par les grands organismes de recherche et chevauchée allègrement par les départements de Recherche et Développement souhaitant bénéficier de la manne publique, en particulier dans les domaines du transport, de l'énergie et de la santé. Avec ce paradigme, l'hyperconnexion de nos infrastructures urbaines associée à un bon usage du «capital intellectuel » réduit à des algorithmes, apporterait des solutions rapides à des problèmes connus et contribuerait directement à la croissance de l'efficacité collective et au bien-être individuel. Mais les rares éléments disponibles permettant de faire un bilan de ces démarches montrent que ce progrès relève d'une mythologie [Cardon, 2015, p. 56-58]. Le rêve d'une intelligence artificielle permettrait certes de réaliser des économies de ressources humaines, mais au prix de services moins efficaces et d'un accroissement massif de la consommation de métaux lourds et surtout d'énergie. Selon l'université de Dresde, l'infrastructure numérique représentait $2 \%$ de l'énergie consommée en 2012 ; elle sera de $50 \%$ en 2033. L'Internet permet de rationaliser l'usage des ressources, mais en même temps, on ne semble pas rationaliser ce que le propre déploiement de l'Internet implique.

15 Les masses d'informations qui peuvent être générées par la captation des traces d'activités en ligne [Boyd et Crawford, 2011; Cardon, 2015] sont également problématiques : qui peut les transformer en «données » exploitables? Qui y a accès? Quelle « valeur » en est tirée sur le plan économique ou politique, à travers les stratégies de prédiction, de prescription, d'objectivation et de transparence? Quelles formes de « gouvernance » cela implique-t-il ?

16 En fait, le déploiement simultané et interdépendant d'horizontalités et de verticalités politiques nouvelles invite à relever le défi épistémologique de penser ensemble ces différents processus d'émancipation et d'aliénation, comme nous y invite Serge Proulx dans son intervention à la journée d'étude ${ }^{8}$ comme dans des publications récentes [Proulx, 2015b]. Et c'est ce défi qui le mène à réinvestir des schèmes critiques de la tradition sociologique pour penser la totalité sociohistorique du capitalisme et de sa version émergente, le «capitalisme informationnel» [Proulx, 2014] ou "capitalisme cognitif $»^{9}$ [Moulier Boutang, 2007; Proulx, 2016], qui se couple avec diverses formes de militantisme pour une société post-capitaliste (logiciel libre, communs de la connaissance, blockchain ${ }^{10}$ ).

Les nouveaux intermédiaires technologiques de nos sociabilités effectuent, via les plateformes, l'appariement entre offre et demande de services, moyennant redevance. Mais la plupart ne contribuent pas aux formes instituées de solidarité, moyennant impôts. Pire, l'ignorance de la diversité des configurations sociotechniques pourrait nous mener à penser que l'Internet est forcément libéral, développant des techniques qui encouragent le processus de désengagement de l'État comme horizon unique, la dérégulation des formes de travail, la propriété non pas collective, mais individuelle, des outils de travail. Là aussi, nous vivons dans une "illusion» ou la terminologie de l'idéologie du partage « apparaît comme une ruse ultime du capitalisme, qui mobilise les valeurs morales pour concentrer de la valeur économique à notre insu ou du moins avec notre consentement tacite » [Proulx, 2015a]. 
18 Le défi d'une approche critique est ainsi de parvenir à saisir ces mécanismes macroscopiques qui opèrent à l'échelle individuelle des comportements nouveaux. Les techniques et services liés à l'Internet savent flatter nos narcissismes, inciter les stratégies de réputation et de reconnaissance au point de promouvoir une addiction générale aux « chiffres de soi » [Sorente, 2011].

\section{Éthique et humanités numériques} des «meilleures pratiques » et de la «neutralité » au regard des contenus véhiculés, ces principes généraux constituent des enjeux de lutte dans la pratique et ne peuvent épuiser la réflexion éthique des acteurs concernés par les réseaux techniques autant que ceux intéressés par le développement des services et des usages. Étant donné l'ampleur des domaines affectés et du pouvoir transformateur que l'on attribue à l'Internet, la réflexion, l'élaboration et l'évaluation de normes éthiques opératoires s'imposent comme une tâche urgente. Il s'agit d'anticiper sur des dérives potentiellement graves même à court terme - s'il n'est pas déjà trop tard, comme le laissent à penser les révélations d'Edward Snowden, autant que le constat alarmant de Zygmunt Bauman : « Nous faisons volontairement beaucoup de choses que les pouvoirs totalitaires cherchaient à imposer par la force et la violence ou la peur » [Bauman, 2013, p. 76]. répondre à cet impératif d'élaborer des référents cognitifs, normatifs ou procéduraux pour encadrer l'innovation et les prises de risques "sociétaux » dans le déploiement d'infrastructures comme de services. La montée en puissance des sciences et technologies de l'information et de la communication (Stic), érigeant la cybernétique en ingénierie sociale, a ainsi mené les systèmes de financement de la recherche européenne à valoriser, voire imposer les contributions interdisciplinaires de nature sociétale. Mais, le plus souvent, cela s'est fait sans définir ce dernier terme, ni même aborder la profondeur philosophique autant que pratique du concept moral de « responsabilité » tel que nous l'a présenté Bernard Reber ${ }^{11}$. De plus, du fait même d'une tradition épistémologique qui impose justement la neutralité axiologique comme précepte déontologique, la grande majorité des disciplines et sous-champs des sciences sociales se trouvent quelque peu désarmés pour répondre à ces attentes et commandes d'un ordre relativement nouveau, les incitant à apposer leur tampon "certifié SHS ", d'une légitimité qui leur semble parfois douteuse.

21 Ce sont plutôt des secteurs nouveaux des SHS, nés à l'intersection de l'innovation industrielle et scientifique, qui tendent plus volontiers à prendre en charge ces missions. Avec le développement des Digital Humanities, les Stic empiètent largement, avec les mathématiciens et les physiciens, dans le champ des objets et des questionnements des sciences humaines et sociales. En tant que sciences appliquées, elles cherchent souvent à apporter des «solutions » sans pour autant procéder à une déconstruction des problèmes ou alors sans prendre en compte le potentiel destructeur des formes instituées et connues de régulation sociale. C'est là tout le débat sur l'ubérisation: une nouvelle technique numérique d'appariement de l'offre et de la demande peut-elle s'autoriser à remettre en cause la structuration décennale quand ce n'est pas centenaire d'un champ d'activité ? Les activités collaboratives doivent-elles être pensées en dehors du droit du travail sédimenté, principalement à l'échelle nationale, par les luttes antérieures? 
22 S'il existe un renouveau des appels à l'interdisciplinarité, notamment entre Stic et SHS, ce ne devrait pas être pour soumettre les SHS aux logiques de prescription qui y règnent (et en particulier la fascination pour la corrélation et la prédiction). Cela devrait au contraire être pour mobiliser des traditions critiques qui relèvent de formations disciplinaires spécifiques et de postures distancées, méthodiquement élaborées par la contemplation du temps long et le recul procuré par le comparatisme. Cette association interdisciplinaire se décline en deux variantes, selon l'environnement d'accueil de cette collaboration : soit les « computerized social sciences" (ou l'instrumentation des sciences sociales et humaines pour la collecte, le traitement et la modélisation d'informations), soit les démarches d'évaluation des paradigmes, dispositifs et configurations développés au sein des Stic et qui fondent la production normative ou prescriptive sur une réflexion ancrée dans l'histoire longue de la philosophie et des humanités.

La contribution des SHS à l'évaluation éthique des risques portés par la production en Stic amène à redéployer le concept philosophique et moral de responsabilité, dans la conception des politiques scientifiques et managériales de l'innovation. Il incombe ainsi aux différentes disciplines et champs thématiques des SHS de contribuer d'une part, à fonder en amont des ontologies sur lesquelles asseoir les procédures d'évaluation. D'autre part, il leur faut alimenter ces ontologies non pas à partir d'un savoir a priori (fondé sur les propriétés du système sociotechnique, ce qui revient à recourir au déterminisme technologique), mais à partir d'une observation des usages proprement dits, d'une description fine des formes d'appropriation de ces techniques qui ne néglige pas l'examen des formes d'émancipation ou de domination qu'elles suscitent. Ainsi, si la sociologie a eu traditionnellement tendance à évacuer la dimension morale, elle doit aujourd'hui s'intéresser à la façon dont sont élaborés les processus de légitimation et de normalisation.

La sociologie contemporaine doit pouvoir fournir des études qui facilitent l'interrogation éthique et l'évaluation morale de l'innovation, comme nous y a invités Pierre-Antoine Chardel $^{12}$, à partir d'un cas précis. En considérant le déploiement des réseaux socionumériques, on peut analyser des formes de sociabilité en ligne, qui affectent les modalités de subjectivation et l'évolution de l'être ensemble par des régimes d'attention, de surveillance et de contrôle des affects. En adoptant le registre de l'évaluation plus que de la prescription, il s'agit alors de mettre en rapport les phénomènes émergents de communication avec les valeurs d'autonomie, de responsabilité, d'attention à l'égard d'autrui, de reconnaissance.

S'il est vrai qu'avec la culture de l'horizontalité, l'influence des pairs s'accroît, d'un autre côté, la culture de la transmission intergénérationnelle, par exemple, l'influence parentale sur les goûts culturels des enfants semble décliner [Pasquier, 2011]. Ce n'est pas seulement la technologie qui doit faire partie de l'évaluation éthique (déterminisme technologique), mais aussi l'environnement social et les relations intersubjectives. C'est ce à quoi Pierre-Antoine Chardel s'est attaché, en étudiant les sociabilités numériques du dispositif d'accompagnement Anamia dans les réseaux "pro-ana» de lutte contre l'anorexie. Sans oublier les logiques de reconnaissance et d'attention qui les sous-tendent (tyrannie de la visibilité), il montre aussi comment se tissent des complémentarités entre blogs, forums et groupes de discussion. L'Internet n'y apparaît finalement pas nocif. Au contraire, des formes de mise en réseau et de solidarité inédites apparaissent pour des individus atteints de pathologies qui avaient tendance à être désocialisantes. Ces logiques spécifiques fonctionnent pour de très nombreux groupes exclus comme les individus 
subissant les déserts médicaux. Mais il reste que les logiques d'appariement proposées par certains algorithmes tendent à consolider l'espace social (clôture des communautés) plutôt qu'à constituer des espaces d'ouverture à l'autre et à d'autres réalités sociales. Elles confortent et approfondissent une vision du monde plus qu'elles ne la confrontent à celle des autres.

\section{Addictions et culture des écrans}

La question de l'addiction aux écrans occupe une part importante des discours médiatiques et domestiques. On pourrait confondre démarche critique et condamnation de l'Internet comme source d'addictions nouvelles, en particulier par la consommation audiovisuelle compulsive, l'usage intensif des médias socionumériques et surtout la pratique apparemment incontrôlée ou incontrôlable des jeux vidéo. Ce jugement de valeur correspond à une forme de résistance qui naît notamment chez les acteurs sociaux les plus menacés ou les plus démunis face au déploiement de nouvelles formes de sociabilité et de pratiques culturelles regroupées sous le vocable de "culture de l'écran ». Ce schisme perceptif vient en grande partie produire un effet générationnel et réifier dans le sens commun, les notions très controversées de « digital natives ", « millennials » ou « génération $Y$ ».

Afin de définir une limite entre us et abus, la posture scientifique critique n'implique pas une condamnation, mais au contraire un dialogue interdisciplinaire pour retracer la genèse sociale des comportements et des jugements de valeur à propos de ces nouvelles pratiques sociales et culturelles. Autant que le déploiement d'enquêtes spécifiques sur la genèse de ces comportements et jugements, un regard rétrospectif sur l'histoire de la réception des technologies de l'information et de la communication semble s'imposer. Car la rhétorique de l'addiction et de l'abrutissement ressurgit régulièrement à propos des médias nouveaux qui émergent, depuis l'apparition du livre de poche jusqu'à celle de la radio et de la télévision.

En ce qui a trait aux comportements, il faut d'abord distinguer la dépendance de l'addiction, pour ensuite discerner à partir des disciplines spécialisées la dimension proprement pathologique des comportements addictifs et enfin, statuer sur son caractère épidémique. En se distanciant des perspectives de sens commun imbues alternativement de technophilie et de technophobie, l'enquête comparative de Nicolas Oliveri ${ }^{13}$ sur la relation fusionnelle aux nouvelles technologies (otakisme) au Japon et en France a ainsi montré l'ancrage culturel des comportements compulsifs dans le jeu vidéo et la diversité de leurs modes de réception.

De son côté, afin d'examiner les propriétés spécifiquement addictives du jeu en ligne, Servane Barrault et Isabelle Varescon ${ }^{14}$ ont comparé des joueurs réguliers de poker en ligne et hors ligne en termes d'intensité de pratique de jeu, de personnalité et de comorbidités psychopathologiques. Le développement des jeux d'argent en ligne (libéralisé très récemment en France) expose les individus plus vulnérables (joueurs à risques) à un dispositif plus accessible, moins socialisé (à domicile) et donnant l'illusion d'un contrôle [Cotte et Latour, 2009]. En évoquant la reconnaissance en psychologie clinique de pathologies spécifiquement liées aux technologies numériques (usage pathologique des jeux vidéo, Internet Gaming Disorder), Pierre Taquet ${ }^{15}$ montre que l'ère numérique implique également la différenciation de savoirs en termes de diagnostics et thérapies comportementale et cognitive en psychologie clinique. Mais la connaissance des 
déterminants de ces pathologies reste très limitée, avec des conséquences importantes pour la détection et l'analyse épidémiologique des différents phénomènes observables. Son enquête sur l'addiction au jeu vidéo permet d'identifier, parmi les joueurs intensifs, ceux qui relèvent de l'addiction pathologique et ceux dont le comportement peut facilement être jugulé dans le cadre de la sociabilité familiale ou à domicile.

\section{Information et accès aux savoirs} produits culturels, autant sur le plan de la production, de la diffusion que de la réception proprement dite. L'accès aux marchés de niche par l'exploitation de la Longue Traîne [Anderson, 2004] ou encore le slogan de la désintermédiation (repris par Creative Commons, par exemple) donnent à croire à la naissance d'un monde radicalement nouveau. L'histoire des techniques de distribution de messages culturels montre qu'il existe un rapport complexe (mais pas une détermination technique simple, cf. Goody, 1979) entre ces techniques, les formes d'organisation de la production et de la diffusion, la genèse de nouveaux formats culturels, et les modalités d'accès et de consommation de ces biens culturels. La spécificité des techniques numériques réside dans le fait qu'elles introduisent simultanément une réorganisation des formes de production, de diffusion et de réception de ces biens culturels [Chartier, 2009].

Dans son exposé à la journée d'étude, Hervé Le $\operatorname{Crosnie}^{16}$ se focalise sur les propriétés socio-économiques spécifiques des réseaux informatiques tels qu'ils se déploient aujourd'hui (non-rivalité des biens numériques, capacité des individus à faire circuler l'information - prenant ainsi la place de professions entières - et coût marginal des biens tendant vers zéro). Ceci pour montrer le poids de ces techniques dans la genèse de nouvelles pratiques de réception, d'appropriation créative et de détournement collaboratif des produits culturels (booktubing, wattpad, fanfiction, fansubbing, machinima, modding ${ }^{17} \ldots$..). Insistant sur les nouvelles formes d'organisation et d'interaction sociale dans la production culturelle, cette approche souligne le rôle créatif et collectif du travail de réception des œuvres, comme le caractère ouvert que peut revêtir la production industrielle qui prend en compte ces pratiques et anticipe sur ce rapport nouveau au public. Cette approche montre aussi la grande diversité et spécificité de ces configurations culturelles, invitant à les analyser pour mieux prendre en compte cette culture numérique émergente dans l'élaboration des politiques publiques et des stratégies industrielles.

Du côté des médias d'information, des formes semblables d'innovation ont attiré l'attention, notamment l'apparition d'une presse totalement numérique, de nouveaux formats journalistiques (blogs) et de réseaux socionumériques (en particulier Facebook et Twitter). Ces formes établies selon des modèles économiques très variables mettent en exergue le rôle nouveau des individus dans la collecte d'information, la multiplication des espaces d'expression de l'opinion et des modalités originales de diffusion et de partage de l'information. Mais le caractère spectaculaire de ces transformations des pratiques médiatiques ne rend pas compte des fortes résistances tant du côté des acteurs hégémoniques de l'industrie culturelle et médiatique, que de celui de la majorité des publics qui s'inscrivent dans une morphologie sociale qui semble évoluer beaucoup plus doucement. Il est donc important de situer les mutations en cours en les intégrant dans le paysage plus général des pratiques d'information. Ainsi, en s'appuyant sur une enquête 
menée entre 2009 et 2012, Rémi Rieffe ${ }^{18}$ nous présente l'évolution des modalités d'accès aux médias d'information d'actualité. L'approche statistique et qualitative ne semble pas indiquer d'évolution brutale dans l'accès à l'information d'actualité, du moins si l'on considère les paramètres sociodémographiques classiques. Les inégalités d'accès aux médias tendent à se reproduire dans les nouveaux médias, et les écarts les plus significatifs concernent principalement les effets de génération (moins de télévision et de presse pour les plus jeunes, selon Sylvie Octobre, 2009).

La posture scientifique et critique doit ainsi relever le défi de prendre en compte à la fois les configurations émergentes et les formes consolidées qui tendent à y résister par le poids des infrastructures économiques, de la morphologie sociodémographique et des habitudes socialement acquises de consommation culturelle et médiatique.

La journée d'étude " us et abus d'Internet » et le présent numéro de Terminal ont ainsi été l'occasion de formuler le défi que représente la réactivation de la posture critique en sciences humaines et sociales pour traiter scientifiquement des questions liées à la pénétration des techniques numériques dans la plupart des sphères d'activité humaine. L'intervention conclusive de Catherine Caleca, maître de conférences en psychologie au CERReV synthétise bien la posture partagée par la diversité des intervenants, et la met en œuvre : l'enjeu est de réinvestir les traditions critiques des humanités au sens large, afin d'établir des repères scientifiquement construits, en tension entre les imaginaires de la révolution et de la simple reproduction des formes de domination, entre les formes d'us et d'abus de et par Internet.

À la façon de Bernard Stiegler (2014) à propos de l'écriture numérique et en référence aux dialogues du Phèdre de Platon, Catherine Caleca convoque en un premier temps le concept du Pharmakón, dont l'usage s'est répandu jusqu'aux conseillers du monde industriel : «(Si) le Web peut être dit pharmacologique, c'est parce qu'il est à la fois un dispositif technologique associé permettant la participation et un système industriel dépossédant les internautes de leurs données pour les soumettre à un marketing omniprésent et individuellement tracé et ciblé par les technologies du user profiling ${ }^{19}$. L'informatique connectée apparaît à la fois comme le remède et le poison, et il faut maintenant être capables d'appréhender dans le même geste, le danger et ce qui sauve.

L'ambivalence des sciences humaines et sociales, quant à Internet, est également évoquée par Catherine Caleca quand elle convoque finalement la figure de Janus, dieu romain représenté avec deux visages, l'un regardant vers le passé et l'autre vers l'avenir. Il incarne le sentiment partagé d'une période de passage angoissante, avec son lot d'héritages et d'opportunités nouvelles, mais aussi un rôle fertilisant de créateur. Comme c'était le cas à l'époque en période de guerre, son temple semble s'être rouvert pour susciter un renouvellement des formes de pensée et d'organisation sociale.

BIBLIOGRAPHIE

Adès, J., Lejoyeux, M. (2001), Encore plus ! Jeu, sexe, travail, argent, Odile Jacob, Paris. 
American Psychiatric Association (2015), DSM 5 : Manuel diagnostic et statistique des troubles mentaux, Masson, Paris.

Anderson, C. (2004), “The Long Tail”, Wired, issue 12.10 [en ligne], Disponible sur : http:// web.archive.org/web/20041127085645/http://www.wired.com/wired/archive/12.10/tail.html, [Consulté le 12 janvier 2016].

Anderson, C. (2008), "The end of theory : the data deluge makes the scientific method obsolete", [en ligne], Disponible sur : http://www.wired.com/2008/06/pb-theory, [Consulté le 12 janvier 2016].

Bauman, Z. (2013), « Une enquête, un entretien, des enjeux », in Au fait, n² 2, juin 2013, p. 76.

Barrault, S. \& Varescon, I. (2016a), "Online and live regular poker players : do they differ in impulsive sensation seeking and gambling practice”, in Journal of Behavioral Addictions, 5(1), pp. 41-50.

Boltanski, L. (2008), Rendre la réalité inacceptable. À propos de la «production de l'idéologie dominante », Demopolis, Paris.

Bourdieu, P. (1994), « Un acte désintéressé est-il possible?», in Raisons Pratiques, Seuil, Paris, pp. 149-167.

Boyd, D. \& Ellison, N., 2007, « Social Network Sites : Definition, History, and Scholarshipin », in Journal of Computer-Mediated Communication, Vol. 13, $\mathrm{n}^{\circ}$ 1, pp. 210-230. [en ligne], Disponible sur : http://onlinelibrary.wiley.com/doi/10.1111/j.1083-6101.2007.00393.x/pdf, [consulté le 3 mars 2016].

Boyd, D. \& Crawford, K. (2011), « Six Provocations for Big Data » [En ligne], Disponible sur : http://papers.ssrn.com/sol3/papers.cfm ?abstract_id=1926431 [consulté le 21 avril 2016].

Cardon, D. (2010), La démocratie Internet. Promesses et limites, Éditions du Seuil, Paris.

Cardon, D. (2015), À quoi rêvent les algorithmes. Nos vies à l'heure des big data, Éditions du Seuil, Paris.

Chardel, P-A. \& Gossaart, C. (dir.) (2012), Conflits des interprétations dans la société de l'information. Éthiques et politiques de l'environnement, Hermès Sciences International, Paris.

Chardel, P-A. (2014), Écologies sociales. Le souci du commun, Parangon, Paris.

Chartier, R. (2009), « L’avenir numérique du livre », in Le Monde [En ligne], Disponible sur : http://www.lemonde.fr/idees/article/2009/10/26/l-avenir-numerique-du-livre-par-rogerchartier_1258883_3232.html, [consulté le 26 janvier 2016].

Combet, N. (2014), « Stiegler, Platon, Derrida. L'écriture/pharmakon », [En ligne], Disponible sur : http://noellecombet.blogspot.fr/2014/01/une-pharmacologie-de-lecriture-stiegler.html, [consulté le 26 janvier 2016].

Cohen-scali, V. \& Guichard J. (2008), «L'identité : perspectives développementales », L'orientation scolaire et professionnelle, 37/3 | 2008, pp. 321-345.

Cotte, J \& Latour, K. (2009). « Blackjack in the kitchen : understanding Online versus Casino gambling », Journal of Consumer Research, 35 : pp. 742-758.

Demunck, J. (2011), « Les trois dimensions de la sociologie critique », in Sociologies [En ligne], Disponible sur : http://sociologies.revues.org/3576, [consulté le 26 janvier 2016].

Denouël, J. \& Granjon, F. (d.), 2011, Communiquer à l'ère numérique : regards croisés sur la sociologie des usages, Transvalor-Presses des Mines, Coll. « Sciences sociales », Paris. 
Elias, N. (1973), La Civilisation des mœurs, Éditions Calmann-Levy, Paris.

Goody, J. (1979), La Raison graphique. La domestication de la pensée sauvage, Éditions de Minuit, Paris.

Granjon, F. (2015), « Du matérialisme comme principium d'un agenda de la recherche critique en communication », in Questions de communication [En ligne], Disponible sur : https :// www.cairn.info/revue-questions-de-communication-2015-2-page-157.htm

Guichard, E. (2012), dir., Regards croisés sur l'internet, ENSSIB, coll. Papiers, Lyon.

Habermas, J. (1962), L'Espace public, Réédition Payot, Paris.

Jouët, J. \& Rieffel, R. (dir.) (2013), S’informer à l'ère numérique, Presses universitaires de Rennes, coll. « RES Public », Rennes.

Jouët, J. (2000), « Retour critique sur la sociologie des usages » [en ligne], Réseaux, n 100, pp. 487-521, Disponible sur : www.persee.fr/doc/reso_0751-7971_2000_num_18_100_2235, [consulté le 26/02/2016].

Lejeune, Y. (2013). TIC 2013, les nouveaux temps réels, Éditions Fy, Paris.

Matthews, J. (2010), « Quelques pistes de réflexion en vue d'une approche critique du Web collaboratif », in Millerand, F., S. Proulx, J. Rueff (dir.), Web social : mutation de la communication, Presses de l'Université du Québec, Québec, pp. 218-227.

Moulier Boutang, Y. (2007), Le Capitalisme cognitif. La Nouvelle Grande Transformation, Éditions Amsterdam, Paris.

Octobre, S. (2009), «Pratiques culturelles chez les jeunes et institutions de transmission : un choc de cultures », in Culture et Prospective [En ligne], Disponible sur : http://www2.culture.gouv.fr/ culture/deps/2008/pdf/Cprospective09-1.pdf, [consulté le 8 avril 2016].

Pasquier, D. (2011), « À cran face aux écrans », in Le Monde Magazine, 26 février 2011, p. 21-27.

Polanyi, K. (1944), La Grande Transformation. Aux origines politiques et économiques de notre temps, Gallimard, Paris.

Proulx, S. (2014), La contribution en ligne. Pratiques participatives à l'ère du capitalisme informationnel, (Direction de l'ouvrage avec J.L. Garcia et L. Heaton), Presses de l'Université du Québec, Québec.

Proulx, S. (2015a), « La sociologie des usages, et après ? », in Revue française des sciences de l'information et de la communication, $n^{\circ} 6$ [En ligne], Disponible sur : http:// rfsic.revues.org/1230, [consulté le 26 février 2016].

Proulx, S. (2015b), « Usages participatifs des technologies et désir d'émancipation : une articulation fragile et paradoxale », in Communiquer, 13 | 2015, pp. 67-77.

Proulx, S. (2016), « La critique du capitalisme cognitif » in F. Aubin, J. Rueff (Eds), Perspectives critiques en communication, Presses de l'Université du Québec, Québec, pp. 191-212.

Revue du MAUSS (2010), « La gratuité - éloge de l'inestimable », n 35, Paris, 1er semestre 2010.

Sorente, I. (2011), Addiction Générale, Éditions J.C. Lattès, Paris.

Souissi, Mohsen. (2013), « La gouvernance de l'internet expliquée concrètement : quels acteurs pour quels rôles ? », in Archives de l'Atelier Internet/ENS, [En ligne], Disponible sur : http:// barthes.ens.fr/atelier/Souissi-Gouvernance-Internet-ENS-2013-04-19.pdf, [consulté le 12 avril 2016]. 
Stiegler, B. (2014) « Pharmacologie du Front National », [En ligne], Disponible sur : http:// noellecombet.blogspot.fr/2014/01/une-pharmacologie-de-lecriture-stiegler.html, [consulté le 20 avril 2016].

Taquet, P. \& Romo, L. (2014), « Les principes de base de la thérapie cognitive et comportementale » in L. Romo M.A. Gorsane (Eds.), Éditions Dunod, Paris, pp. 82-101.

Tisseron, S. (2012), «Culture numérique : une triple révolution, culturelle, cognitive et psychique » [En ligne], Disponible sur : http://www.sergetisseron.com/blog/nouvel-article-618, [consulté le 25 octobre 2016].

\section{NOTES}

1. La journée d'étude a été organisée à l'université de Caen-Normandie, le 25 septembre 2015 par le CERReV, avec le concours de la MRSH, de l'IUT de Caen et de l'équipe MADYNES/Inria. Les enregistrements des communications sont accessibles sur Canal $U$ à cette adresse: https:// www.canal-u.tv/producteurs/centre_d_enseignement_multimedia_universitaire_c_e_m_u/ $\mathrm{mrsh} /$ cerrev/us_et_abus_d_internet

2. Voir, à ce propos, la journée d'étude SFIC/Cnam du 30 mars 2016 sur « Digital Studies et SIC divergences ou complémentarités?»

3. Le crowdsourcing (ou myriadisation du travail) est une forme d'externalisation ouverte ou de collaboration participative faisant appel à la contribution libre d'individus à l'extérieur d'une organisation, qu'elle soit entreprise, service public ou tiers secteur, par l'intermédiaire de plateformes dédiées. La plus connue des ces plateformes est Amazon Mechanical Turk (AMT).

4. Le crowfunding ou financement participatif est une autre façon pour les organisations ou les particuliers de collecter des fonds pour leurs projets par l'intermédiaire de plateformes en ligne.

5. Le crowdsensing est une technique où un groupe d'individus partagent collectivement les données des capteurs de leurs terminaux informatiques pour permettre l'élaboration de mesures, de cartes, d'analyses, d'estimations ou de prédiction de processus le plus souvent présentés comme étant d'intérêt commun.

6. «La baguette magique du tout-connecté " par Évelyne Bourdoux, maître de conférences en Sciences de l'information et de la communication à l'INTD/Cnam, entretien dans ce numéro.

7. La smart city ou ville intelligente désigne une ville qui exploite la technologie et l'innovation pour rendre plus efficace l'utilisation des ressources et réduire notamment la taille de l'empreinte écologique.

8. « Enjeux sociaux de la participation en ligne : entre marchandisation et partage».

9. Cf. Moulier Boutang Yann (2007), Le Capitalisme cognitif. La Nouvelle Grande Transformation, éd. Amsterdam, Paris, 2007.

10. Blockchain est une série de technologies permettant le stockage et la transmission d'informations de façon sécurisée et décentralisée. Cette ressource issue la cryptographie est ainsi à la base de la monnaie bitcoin.

11. "Humanités numériques responsables " par Bernard Reber, directeur de recherche au CNRS, Centre de recherches politiques (Cevipof) de Sciences Po Paris, dans ce numéro.

12. «Les réseaux numériques comme source d'interrogation éthique " par Pierre-Antoine Chardel, professeur de philosophie sociale et d'éthique à Télécom École de Management, communication à la journée d'étude "Us et abus de l'Internet".

13. "Cyberdépendance: analyse comparative France-Japon " par Nicolas Oliveri, professeur de philosophie sociale et d'éthique à Télécom École de Management et directeur de recherches à l'université Paris-Descartes, dans ce numéro. 
14. «L'addiction aux jeux de hasard et d'argent en ligne : quelles spécificités? " par Servane Barrault, maitre de conférences en Psychologie, université François-Rabelais de Tours \& Isabelle Varescon, professeur de Psychologie Clinique et Psychopathologie, Institut Universitaire de Psychologie Paris Descartes, dans ce numéro.

15. «Les motivations dans l'usage pathologique des jeux vidéo : théories et thérapie " par Pierre Taquet, psychologue clinicien (Groupe Hospitalier Seclin Carvin), docteur en psychologie, associé au Laboratoire PSITEC-EA 4072 à l'Université Lille 3 et chercheur au CHRU de Lille à l'Hôpital Fontan II, dans ce numéro.

16. «CEuvres transformatives numériques : de la culture lettrée aux pratiques collaboratives » par Hervé Le Crosnier, maître de conférences en Sciences de l'information et de la communication à l'Université de Caen Normandie, communication à la journée d'étude "us et abus de l'Internet".

17. Le Booktubing désigne la production de chroniques littéraires qui sont ensuite publiées sur les plateformes de streaming vidéo; Wattpad est une plateforme d'écriture et de partage de productions littéraires; le terme fanfiction désigne des récits dérivés de produits médiatiques tels que films, séries, romans, mangas ; le fansubbing est la pratique de sous-titrage collaboratif de copies illégales de films; machinima est un néologisme désignant des fictions vidéos élaborées à partir de séquences vidéo capturées en temps réel (le plus souvent des jeux vidéos) ; le modding est la pratique de création de jeux vidéos à partir des modifications du jeu original.

18. "Us et abus... pour le sociologue, la réalité sociale est grise", par Rémy Rieffel, sociologue des médias, professeur à l'université Paris II Panthéon-Assas et à l'Institut français de presse (IFP) et directeur du Carism, entretien dans ce numéro.

19. Introduction d'Ars Industrialis, Association internationale pour une politique industrielle des technologies de l'esprit (http://www.arsindustrialis.org/pharmakon).

\section{RÉSUMÉS}

Les technologies numériques touchent désormais l'ensemble des secteurs de la vie sociale, interrogeant les certitudes et les paradigmes économiques, juridiques, technologiques et sociétaux établis. Plongées dans les transformations engendrées par des technologies numériques puissamment adossées à une idéologie du progrès, les sciences humaines et sociales ne sont pas toujours parvenues à trouver une posture permettant de développer une réflexion structurée capable de les comprendre. Face à ces mutations ce dossier propose plusieurs approches critiques, pour faire le bilan de leurs pratiques.

The digital technologies now affect all sectors of social life, questioning established economic, legal, technological and societal certainties and paradigms. Immersed into the transformations engendered by digital technologies that are powerfully backed by an ideology of progress, the human and social sciences have not always succeeded in finding a posture to develop a structured reflection capable of understanding them. Faced with these changes, this dossier proposes several critical approaches, to make an assessment of their practices. 
INDEX

Keywords : Epistemology, criticism, Human and Social Sciences, Internet, digital.

Mots-clés : Épistémologie, critique, sciences humaines et sociales, Internet, numérique.

\section{AUTEURS}

\section{CÉCILE DOLBEAU-BANDIN}

26, rue du Saint-Graal 14000 Caen

0686640752

Unicaen (France), CERReV

cecile.dolbeau@gmail.com

\section{SERGE PROULX}

Université du Québec à Montréal / École des médias

488, rue Duvernay

Longueuil, Canada J4K 4K7

14504632366

proulx.serge@uqam.ca

\section{VASSILI RIVRON}

MADYNES / Inria

615 , rue du Jardin Botanique

54600 Villers-lès-Nancy

0650832984

vassili.rivron@inria.fr 